\title{
SOLVING THE UHLMANN EQUATION FOR THE BURES- FISHER METRIC ON THE SUBSET OF RANK-DEFICIENT QUDIT STATES
}

\author{
M. Bures ${ }^{1,2, a}$, A. Khvedelidze ${ }^{2,3,4}$, D. Mladenov ${ }^{5}$ \\ ${ }^{1}$ Institute of Experimental and Applied Physics Czech Technical University in Prague, \\ Husova 240/5 11000 Prague 1. Czech Republic \\ ${ }^{2}$ Meshcheryakov Laboratory of Information Technologies, Joint Institute for Nuclear \\ Research, Dubna, Russia \\ ${ }^{3}$ A.Razmadze Mathematical Institute, Iv.Javakhishvili Tbilisi State University, Tbilisi, \\ Georgia \\ ${ }^{4}$ Institute of Quantum Physics and Engineering Technologies, Georgian Technical \\ University, Tbilisi, Georgia \\ ${ }^{5}$ Faculty of Physics, Sofia University “St. Kliment Ohridski”, 5 James Bourchier Blvd, 1164 Sofia, \\ Bulgaria \\ E-mail: ${ }^{a}$ bures@physics.muni.cz
}

The Bures-Fisher metric on the subset $\mathfrak{P}_{N, k}$ of the state space of an $N$-level quantum system, consisting of rank- $k$ density matrices is given by a solution to the Uhlmann equation. Solving the Uhlmann equation $\mathfrak{P}_{N, k}$ on, we use its $\mathfrak{P}_{N, k}=\bigcup_{\alpha} \mathfrak{P}_{\left[H_{\alpha}\right]}^{k}$ decomposition into a finite union of strata $\mathfrak{P}_{\left[I I_{\alpha}\right]}^{k}$ of different $S U(N)$ orbit types with all admissible isotropy groups $H_{\alpha}$. Solution to the Uhlmann equation on the corresponding orbits stratum $\mathfrak{P}_{\left[H_{\alpha}\right]}^{k}$ defines uniquely the Bures-Fisher metric for a rank deficient states.

Keywords: quantum computing, quantum information, quantum Fisher information, Bures metric, qubit, qutrit, qudit

Martin Bures, Arsen Khvedelidze, Dimitar Mladenov 


\section{Introduction}

Modern developments in theoretical quantum metrology have given rise to a fresh interest in the quantum Fisher information and the corresponding Riemannian geometry structure, the Bures metrics on the state space of a finite dimensional quantum system (see e.g. the review in [1] and references therein). Currently, it is a common view that:

- the Bures metric is locally equivalent to a Riemannian metric determined by the quantum analog of the Fisher information matrix [2, p.262];

- the quantum Fisher information matrix and the Bures metric are equivalent to each other, except at the points where the rank of the density matrix changes [3-6];

To clarify these interrelations, the knowledge of generic topological features and differentialgeometrical properties of the convex body of quantum states is very useful. More precisely, aiming to determine the Bures-Fisher metric on the subset of fixed rank- $k$ states, $\mathfrak{P}_{N, k}$, it is helpful to decompose it into components of strata of orbits of adjoint action of the unitary group. This decomposition follows by combining two partitions of the state space $\mathfrak{P}_{N}$ into different topological subspaces. The first one is a well-defined partition, the stratification of $\mathfrak{P}_{N}$ into unitary orbit types:

$$
\mathfrak{P}_{N}=\bigcup_{\text {orbit types }} \mathfrak{P}_{\left[H_{\alpha}\right]}
$$

where $\mathfrak{P}_{\left[H_{\alpha}\right]}:=\left\{\varrho \in \mathfrak{P} \mid\right.$ isotropy group of $\varrho$ is conjugate to $\left.H_{\alpha}\right\}$ is the stratum associated to the isotropy group $H_{\alpha}$. The second partition is the decomposition of the state space $\mathfrak{P}_{N}$ into subsets

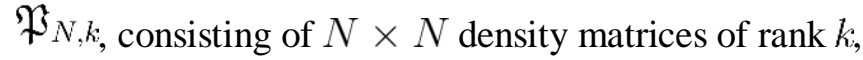

$$
\mathfrak{P}_{N}=\bigcup_{\text {ranks }} \mathfrak{P}_{N, k}
$$

Comparing (1) and (2), we arrive at the decomposition of each component $\mathfrak{P}_{N, k}$ of a fixed rank-k as a union of orbits of certain types,

$$
\mathfrak{P}_{N, k}=\bigcup_{\text {orbit types }} \mathfrak{P}_{\left[H_{\alpha}\right]}^{k}
$$

In the next section, we briefly summarize the results of studying the Uhlmann equation (4) on each component of (3). It will be outlined that the knowledge of the topological decomposition (3) and the unique Bures-Fisher metric $g_{\left[I I_{\alpha}\right]}$ on unitary strata allows to define the corresponding metric $g_{N, k}$ on rank-k subset $\varrho \in \mathfrak{P}_{N, k}$ and analyze the singularities of this metric in the neighbourhood of states with different ranks. 


\section{Bures-Fisher metric from the Uhlmann equation}

Let $\varrho$ be an element of $\mathfrak{P}_{N, k}$, a set of unit trace semi-positive $N \times N$ density matrices of rank $k$. For a given $\varrho$, consider the equation

$$
\varrho \mathrm{G}_{\varrho}+\mathrm{G}_{\varrho} \varrho=\mathrm{d} \varrho
$$

for an unknown 1-form $\mathrm{G}_{\varrho}$. According to A.Uhlmann [7], the solution to (4) determines the BuresFisher metric on $\mathfrak{P}_{N, k}$ as

$$
\mathrm{g}=\frac{1}{2}\left(\mathrm{~d} \varrho, G_{\varrho}\right)
$$

In order to treat $\mathfrak{P}_{N, k}$ as a Riemannian manifold endowed with the Bures-Fisher metric, we need to analyze the existence and uniqueness of the solution to (4) for all $k=1,2, \ldots, N$. Following the standard theory of systems of linear equations, one can easily formulate the corresponding conditions on the density matrix, which guarantee the existence and uniqueness of the Bures-Fisher metric (5). The Uhlmann equation, being a system of linear equations, has its solution represented as

$$
\mathrm{G}_{\varrho}=\mathrm{G}_{\varrho}^{(0)}+\mathrm{G}_{\varrho}^{(p)},
$$

where $\mathrm{G}^{(p)}$ is some particular solution and $\mathrm{G}^{(0)}$ stands for a general solution of the corresponding homogeneous equation. If $\operatorname{rank}(\varrho)=N$ then $\mathrm{G}_{\varrho}^{(0)}$ is trivial, while for singular density matrices, i.e. rank deficient states with $k=\operatorname{rank}(\varrho) \leq N-1$, the number of linearly independent solutions of the homogeneous Uhlmann equation is $(N-k)^{2}$. However, it can be shown that the metric form (5), evaluated at the vectors tangent to $\mathfrak{P}_{N, k}$ turns out to be independent of all $(N-k)^{2}$ parameters in the solution to the Uhlmann equation. To verify these statements, we note that equation (4) is a special form of the famous Sylvester matrix equation with matrices $\left.A \in \mathrm{M}_{m \times m}(\mathbb{C}), B \in \mathrm{M}_{n \times n}(\mathbb{C}), C \in \mathrm{M}_{m \times n}(\mathbb{C})\right)$ for an unknown $m \times n$ matrix $X:^{1}$

$$
A X+X B=C .
$$

In 1884, Sylvester (cf.[8]) considered the homogeneous version of this equation and thereby showed that the condition for (7) to have a unique solution is that $A$ and $-B$ have no eigenvalues in common. Following these propositions for equal Hermitian matrices $A=B=A^{\dagger}$, one can prove that

a) equation (4) admits the Bures-Fisher metric on states of all possible ranks;

b) for rank deficient states, rank $(\rho)=k<N$, the solution to (4) is not unique, but the Bures-Fisher metric (5) on every singular stratum is unique iff all directions of the variation $\mathrm{d} \rho$ are tangent to the hypersurface of fixed rank matrices;

c) the SVD decomposition of density matrices on each component $\mathfrak{P}_{\left[H_{\alpha]}\right]}^{k}$ in (3) allows for an explicit solution to the Uhlmann equation to be written and define the Bures-Fisher metric on the product of the generalized flag manifold $S U(N) / H_{\alpha}$ and $k$-simplex.

\section{Concluding remarks: Bures-Fisher metric on qubit fixed rank states}

A detailed proof and explicit formulae for the generic case will be presented elsewhere. Here we only state results for the case of a 2-level system, a single qubit.

For a single qubit, there are two types of $S U(2)$-orbits, labeled by the corresponding isotropy 
group $\left[H_{\alpha}\right], \alpha=0,1$, respectively. For the maximally mixed state, $H_{0}=S U(2)$, and for generic states, $H_{1}=S(U(1) \times U(1)) \in S U(2)$, up to conjugation. The sought-for orbit types, the stratification (1) of the single qubit state space $\mathfrak{P}_{2}$ is given by the following components:

$$
\begin{gathered}
\text { (max. mixed stratum) } \quad \mathfrak{P}_{\left[H_{0}\right]}:=\left\{\varrho=\frac{1}{2} \mathbb{I}\right\} \\
\text { (generic stratum) } \quad \mathfrak{P}_{\left[H_{1}\right]}:=\left\{\varrho \in \mathfrak{P}_{2} \mid 0>r_{1}>r_{2}>1\right\} .
\end{gathered}
$$

Hence, the components of the partition $\mathfrak{P}_{2}=\mathfrak{P}_{2,2} \bigcup \mathfrak{P}_{2,1}$ with respect to the rank are:

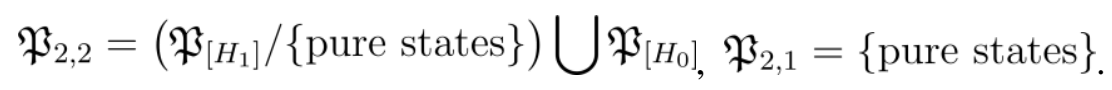

Using the SVD decomposition of a qubit state, $\varrho=U \operatorname{diag}\left(r_{1}, r_{2}\right) U^{\dagger}$, with the unitary factor $U$ in the Euler (3-2-3)-parameterization by angles $(\alpha, \beta, \gamma)$, the solutions to the Uhlmann equation for the corresponding Bures-Fisher metric are:

$$
\begin{gathered}
\mathrm{g}_{\left[H_{0}\right]}=\frac{1}{2}\left(\sin ^{2} \beta(\mathrm{d} \alpha)^{2}+(\mathrm{d} \beta)^{2}\right), \\
\mathrm{g}_{\left[H_{1}\right]}=\frac{1}{2} \frac{(\mathrm{d} r)^{2}}{1-r^{2}}+\frac{1}{2} r^{2}\left(\sin ^{2} \beta(\mathrm{d} \alpha)^{2}+(\mathrm{d} \beta)^{2}\right),
\end{gathered}
$$

where $r=r_{1}-r_{2}$ denotes the Bloch radius of a qubit. Now passing to the new coordinate $\chi=\arcsin (r)$, we identify the maximal rank stratum $\mathfrak{P}_{2,2}$ with the Uhlmann 3-hemisphere (cf. [9]) of radius $1 / \sqrt{2}$, embedded in a standard way into the Euclidean space $\mathbb{R}^{4}$ and the Bures-Fisher metric is identical to the induced metric:

$$
\mathrm{g}_{2,2}=\frac{1}{2}\left((\mathrm{~d} \chi)^{2}+\sin ^{2} \chi\left(\sin ^{2} \beta(\mathrm{d} \alpha)^{2}+(\mathrm{d} \beta)^{2}\right)\right), \quad \chi \in\left[0, \frac{\pi}{2}\right),
$$

while the Bures-Fisher metric $g_{2,1}$ on rank-1 states, $\left(\chi=\frac{\pi}{2}\right)$, is the standard round metric on the 2sphere $\mathbb{S}_{2}\left(\frac{1}{\sqrt{2}}\right) \subset \mathbb{S}_{3}\left(\frac{1}{\sqrt{2}}\right)$. 


\section{Acknowledgement}

This work was supported in part by the "Bulgaria-JINR" and "Czech Republic-JINR" Collaborative grants. Additionally, the work of MB was supported in part by the EU Regional Development Fund-Project No. CZ.02.1.01/0.0/0.0/16019/0000766. AK acknowledges the financial support of the Shota Rustaveli National Science Foundation of Georgia, Grant FR-19-034. DM was supported in part by the Bulgarian National Science Fund research grant DN 18/3.

\section{References}

[1] Jing Liu et al, Quantum Fisher information matrix and multiparameter estimation, J. Phys.A: Math. Theor. 53023001 (2020)

[2] Holevo, Alexander S, Probabilistic and Statistical Aspects of Quantum Theory, Dordrecht: Springer (2011)

[3] D. Safranek, Discontinuities of the quantum Fisher information and the Bures metric, Physical Review A95, 052320 (2017)

[4] Sisi Zhou, Liang Jiang, An exact correspondence between the quantum Fisher information and the Bures metric, arXiv: $1910.08473 \mathrm{v} 1$

[5] A.T.Rezakhani, M.Hassani, and S.Alipour, Continuity of the quantum Fisher information, Phys. Rev. A 100, 032317, (2019)

[6] L.Seveso et al., On the discontinuity of the quantum Fisher information for quantum statis-tical models with parameter dependent rank, J. Phys.A: Math. Theor. 53 02LT01 (2020)

[7] Armin Uhlmann, Geometric Phases and Related Structures, Rep. Math. Phys., 36461-481, (1995)

[8] C.C.MacDuffee, The theory of matrices, Chelsea (1956)

[9] J.Dittmann, On the Riemannian Geometry of Finite Dimensional Mixed States, Seminar Sophus Lie 3, 73-87, (1993). 